\title{
Is robot-assisted partial nephrectomy safe for high complexity tumors?
}

\author{
Periklis Koukourikis, Ali Abdullah Alqahtani, Koon Ho Rha \\ Department of Urology and Urological Science Institute, Yonsei University College of Medicine, Seoul, Korea \\ Correspondence to: Dr. Koon Ho Rha. Professor of Urology, Department of Urology and Urological Science Institute, Yonsei University College of \\ Medicine, Severance Hospital, Yonseiro 50-1, Seodaemun-gu, Seoul 120-752, Korea. Email: khrha@yuhs.ac. \\ Comment on: Buffi NM, Saita A, Lughezzani G, et al. Robot-assisted partial nephrectomy for complex (PADUA score $\geq 10$ ) tumors: techniques and \\ results from a multicenter experience at four high-volume centers. Eur Urol 2020;77:95-100.
}

Submitted Mar 30, 2020. Accepted for publication May 29, 2020.

doi: $10.21037 /$ tau-20-836

View this article at: http://dx.doi.org/10.21037/tau-20-836

Nephron-sparing surgery (NSS) is the treatment of choice for cT1 kidney tumors as it provides similar oncological safety in comparison with radical nephrectomy with the advantage of preservation of renal function and improved overall survival (1). In the minimally invasive era, robotassisted partial nephrectomy (RAPN) has gained great popularity, when compared with open or laparoscopicassisted NSS, as it offers similar oncological results with better perioperative outcomes (2,3). Furthermore, the robotic approach offers to surgeon three-dimensional (3-D) visualization of the operative field, improved dexterity as well as precision of movements resulting in a shorter learning curve. RAPN has become the preferred approach over laparoscopic-assisted partial nephrectomy even for large and complicated tumors.

The article by Buffi et al., which was published in a recent issue of European Urology, explored the outcomes of RAPN in patients with complex renal tumors (PADUA score $\geq 10$ ) and compared the observed outcomes between patients with increasing complexity. The authors, also, described the use of the latest induced technologies in RAPN, such as the TilePro technology, near-infrared fluorescence (NIRF) imaging, and hyperaccuracy 3-D (HA3D) reconstruction. In their retrospective analysis, they included 225 patients, and the surgeries were performed in four different tertiary centers over a period of 7 years. Within the cohort, 111 patients had a PADUA score of 10,85 patients had a PADUA score of 11 , while 45 and 14 patients had a PADUA score of 12 and 13 , respectively.

The authors have correctly identified the lack of large, multi-institutional studies in the literature assessing the outcomes of RAPN in patients with highly complex tumors and attempted to elucidate this specific field. The primary outcome of this study was the reporting of the outcomes of RAPN in the selected patient population. The approach was transperitoneal in $186(72.9 \%)$ patients and retroperitoneal in 69 patients $(27.1 \%)$ and the median operative time was 159 (IQR: 120-210) minutes. The type of ischemia was main artery clamping to the majority of patients, while selective clamping and zero ischemia were also used ( $70.4 \%$ vs. $16.5 \%$ vs. $5.1 \%$, respectively). A median estimated blood loss of 150 (IQR: 100-250) mL and a median warm ischemia time (WIT) of 18 (IQR: 15-23) were noted. Authors reported a Clavien-Dindo $>2$ complication in $13(5.1 \%)$ patients, while positive surgical margins (PSMs) were observed in 4 patients $(1.9 \%$ of patients with malignant histology), and the conversions to open surgery and radical nephrectomy were $4(1.6 \%)$ and 5 $(1.9 \%)$, respectively.

An additional outcome was the achievement of optimal surgical outcomes, which was defined according to the Margin Ischemia and Complication (MIC) binary system (absence of Clavien-Dindo $>2$ complications, WIT $<20 \mathrm{~min}$, and absence of PSMs) (4). Overall, optimal surgical outcomes were achieved in 158 (62\%) patients. Regarding tumors with the highest complexity, the results showed a significantly lower rate of MIC achievement in tumors of PADUA score 12-13 compared to tumors of PADUA score 10 and PADUA score 11, 40.7\%, 68.5\%, and 68.2\%, respectively $(\mathrm{P}=0.001)$. Independent predictors of not 
achieving MIC in multivariate analysis were PADUA score 12-13 and male gender. These results are in line with previous studies; in a large single institutional series, the rate of optimal surgical outcomes was decreasing through the patients as the PADUA complexity score was increasing, from $65.3 \%$ and $56.9 \%$ for patients with low and intermediate complexity tumors to $37.5 \%$ for patients with high complexity tumors (5). In a recent paper, Takahara et al. noted a lower rate of optimal surgical outcomes achievement in the high-complexity group (PADUA $\geq 10$ ) of patients compared to non-high-complexity group (68.3\% vs. $86.3 \%$, respectively, $\mathrm{P}=0.009$ ) (6).

Taking into account the three different outcomes included in MIC achievement between the groups of patients, in this analysis, the main cause of failure for optimal surgical outcomes was increased ( $>20$ minutes) WIT. WIT was 21 (IQR: 18-27) minutes in patients with PADUA 12-13 compared to 17 (IQR: 14-21) minutes in patients with PADUA $10(\mathrm{P}<0.001)$, with more patients at highest complexity group having a WIT $>20 \mathrm{~min}$ (39.7\% vs. $15.7 \%, \mathrm{P}=0.015$, PADUA $12-13$ vs. PADUA 10 , respectively). On the other hand, no significant difference was found between the groups of patients with lower and higher complexity tumors on PSMs and major complications (Clavien-Dindo $>2$ ) rates. WIT remains a topic of interest and debate in the literature, as the duration of ischemia is the only modifiable surgical factor affecting kidney function preservation, while the other factors are patient-depended and unmodifiable (age, preoperative renal function, comorbidities). A proposed acceptable time limit is 20-30 minutes, but most authors agree that it is not a binary outcome and that every minute counts during hilar clamping $(7,8)$. Previous data, also, support the correlation between high PADUA score and increased WIT $(9,10)$. The prolongation of ischemia duration seems a natural consequence during RAPN in complicated tumors as the reconstruction of the large collecting system and renal parenchyma defect have to be performed meticulously to avoid complications such hemorrhage and urine leakage.

We have to move beyond WIT analysis, studies support that prolonged WIT has a negative impact on functional outcomes, although they have focused mainly in the shortterm period (11). We support the idea that WIT has only a minor effect in long-term functional outcomes in patients with a normal contralateral kidney. The results of our series showed that neither WIT nor the method of clamping significantly affects the estimated glomerular filtration rate $(\mathrm{eGFR})$ in a long-term period of follow-up $(5,12)$. Even in cases of extreme WIT prolongation (30-60 min) there are data to support the mild structural changes on human kidney and the minimum effect on long-term functional outcomes $(13,14)$. Authors choose to use the MIC achievement, which is a standardized system for reporting optimal surgical outcomes for NSS, to report and compare their results. However, this system does not include analysis of long-term functional outcomes and a separate analysis of how the difference in WIT between the groups of patients affected the long-term functional outcomes were not performed in this study. Recently, a new system of trifecta outcomes reporting for RAPN was published by Brassetti et al., which excludes WIT and includes postoperative eGFR reduction (15). This new reporting system could be more suitable for assessing RAPN outcomes in series where different techniques for hilar clamping are used, such as selective artery clamping or zero ischemia.

We must be grateful to authors for presenting the use of novel technologies, undoubtedly they are of great importance for assisting the surgeon to perform more complex cases. However, authors did not report the impact of the use of these techniques on perioperative outcomes and MIC achievement in this cohort. Also, always we have to bear in mind the economic impact for their use and difficulties adopting them in everyday practice. In our clinical practice, as in most institutions performing RAPN, the use of intraoperative laparoscopic ultrasound combined with TilePro technology is essential for almost every RAPN. Furthermore, we have previously described a simplified zero ischemia technique using kidney donor computed tomographic angiography, TilePro and conventional laparoscopic bulldog clamps, which is an easily adopted technique without special requirements (16). We believe that the use of novel technologies should be further assessed and validated in future prospective and ideally randomized studies, evaluating the oncological and the functional outcomes of RAPN.

The present study encounters some limitations. The retrospective nature of this study is a major drawback, albeit we have to admit that the inclusion of patients from different institutions increased the external validity of the study. The lack of definite inclusion criteria between different surgeons induced selection bias and the use of different surgical techniques and technologies during the period of study increase the heterogeneity, and authors have recognized it. The median follow-up period was relatively 
short (28 months) for oncological outcomes reporting; this period of follow-up is adequate for long-term functional outcomes reporting, whereas authors did not include this outcome in the analysis.

RAPN is a feasible and effective treatment, when performed by experienced surgeons in accordance with the use of the novel technologies, even for the most challenging cases. However, in tumors with the highest complexity (PADUA 12-13), the surgical outcomes may be suboptimal and proper patient counseling should be given. In light of these promising results, there are indications that the highrisk group suggested by PADUA classification seems to be not a single category. Further studies are needed to define the proper risk stratification and assessment of oncological and functional outcomes to safely extend the indications for RAPN.

\section{Acknowledgments}

Funding: None.

\section{Footnote}

Provenance and Peer Review: This article was commissioned by the editorial office, Translational Andrology and Urology. The article did not undergo external peer review.

Conflicts of Interest: All authors have completed the ICMJE uniform disclosure form (available at http://dx.doi. org/10.21037/tau-20-836). KHR serves as an unpaid editorial board member of Translational Andrology and Urology from Jun 2017 to May 2021. The other authors have no conflicts of interest to declare.

Ethical Statement: The authors are accountable for all aspects of the work in ensuring that questions related to the accuracy or integrity of any part of the work are appropriately investigated and resolved.

Open Access Statement: This is an Open Access article distributed in accordance with the Creative Commons Attribution-NonCommercial-NoDerivs 4.0 International License (CC BY-NC-ND 4.0), which permits the noncommercial replication and distribution of the article with the strict proviso that no changes or edits are made and the original work is properly cited (including links to both the formal publication through the relevant DOI and the license). See: https://creativecommons.org/licenses/by-nc-nd/4.0/.

\section{References}

1. Kunath F, Schmidt S, Krabbe LM, et al. Partial nephrectomy versus radical nephrectomy for clinical localised renal masses. Cochrane Database Syst Rev 2017;5:CD012045.

2. Peyronnet B, Seisen T, Oger E, et al. Comparison of 1800 robotic and open partial nephrectomies for renal tumors. Ann Surg Oncol 2016;23:4277-83.

3. Choi JE, You JH, Kim DK, et al. Comparison of perioperative outcomes between robotic and laparoscopic partial nephrectomy: a systematic review and metaanalysis. Eur Urol 2015;67:891-901.

4. Buffi N, Lista G, Larcher A, et al. Margin, ischemia, and complications (MIC) score in partial nephrectomy: a new system for evaluating achievement of optimal outcomes in nephron-sparing surgery. Eur Urol 2012;62:617-8.

5. Abdel Raheem A, Alatawi A, Kim DK, et al. Outcomes of high-complexity renal tumours with a Preoperative Aspects and Dimensions Used for an Anatomical (PADUA) score of $\geq 10$ after robot-assisted partial nephrectomy with a median 46.5-month follow-up: a tertiary centre experience. BJU Int 2016;118:770-8.

6. Takahara K, Sumitomo M, Fukaya K, et al. Predictors for trifecta achievement of robot-assisted partial nephrectomy in high-complexity tumors (Preoperative Aspects and Dimensions Used for an Anatomical score $\geq 10$ ). Asian J Endosc Surg 2020;13:390-6.

7. Becker F, Van Poppel H, Hakenberg OW, et al. Assessing the impact of ischaemia time during partial nephrectomy. Eur Urol 2009;56:625-34.

8. Desai MM, Gill IS, Ramani AP, et al. The impact of warm ischaemia on renal function after laparoscopic partial nephrectomy. BJU Int 2005;95:377-83.

9. Lee JW, Cho SY, Jeon C, et al. The association between the anatomical features of renal tumours and the functional outcomes of robot-assisted partial nephrectomy. Can Urol Assoc J 2014;8:E810-4.

10. Ficarra V, Bhayani S, Porter J, et al. Predictors of warm ischemia time and perioperative complications in a multicenter, international series of robot-assisted partial nephrectomy. Eur Urol 2012;61:395-402.

11. Dagenais J, Maurice MJ, Mouracade P, et al. The synergistic influence of ischemic time and surgical precision on acute kidney injury after robotic partial nephrectomy. Urology 2017;107:132-7.

12. Komninos C, Shin TY, Tuliao P, et al. Renal function is the same 6 months after robot-assisted partial nephrectomy 
regardless of clamp technique: analysis of outcomes for off-clamp, selective arterial clamp and main artery clamp techniques, with a minimum follow-up of 1 year. BJU Int 2015;115:921-8.

13. Parekh DJ, Weinberg JM, Ercole B, et al. Tolerance of the human kidney to isolated controlled ischemia. J Am Soc Nephrol 2013;24:506-17.

14. Bhayani SB, Rha KH, Pinto PA, et al. Laparoscopic partial nephrectomy: effect of warm ischemia on serum creatinine.

Cite this article as: Koukourikis $\mathrm{P}$, Alqahtani AA, Rha KH. Is robot-assisted partial nephrectomy safe for high complexity tumors? Transl Androl Urol 2020;9(6):2455-2458. doi: 10.21037/ tau-20-836
J Urol 2004; 172:1264-6.

15. Brassetti A, Anceschi U, Bertolo R, et al. Surgical quality, cancer control and functional preservation: introducing a novel trifecta for robot-assisted partial nephrectomy. Minerva Urol Nefrol 2020;72:82-90.

16. Shin TY, Choi KH, Lim SK, et al. Simplified zero ischemia in robot assisted partial nephrectomy: initial yonsei experience. Korean J Urol 2013;54:78-84. 